\title{
OPTIMIZACIÓN DE LAS CONDICIONES ANALÍTICAS IDEALES PARA CUANTIFICAR ALUMINIO EN TEJIDOS DE RATAS SPRAGUE DAWLEY MEDIANTE LA TÉCNICA DE ABSORCIÓN ATÓMICA
}

\author{
Alejandro Monserrat GARCÍA ALEGRÍA ${ }^{1 *}$, Agustín GÓMEZ-ÁLVAREZ², Iván ANDURO-CORONA³, \\ Armando BURGOS-HERNÁNDEZ ${ }^{4}$, Eduardo RUÍZ-BUSTOS ${ }^{1}$, Rafael CANETT-ROMERO ${ }^{4}$ y \\ Humberto Francisco ASTIAZARÁN-GARCÍA ${ }^{1,3}$
}

\footnotetext{
${ }^{1}$ Programa de Doctorado en Ciencias (Químico Biológicas y de la Salud). Universidad de Sonora. Luis Encinas y Rosales. Hermosillo, Sonora, C. P. 83000.

2 Departamento de Ingeniería Química y Metalurgia. Universidad de Sonora. Luis Encinas y Rosales. Hermosillo, Sonora, C. P. 83000.

${ }^{3}$ Centro de Investigación en Alimentación y Desarrollo, A.C. Dirección de Nutrición. Carretera a la Victoria, kilómetro 0.6. Hermosillo, Sonora, México, C. P. 83304.

${ }^{4}$ Departamento de Investigación y Posgrado en Alimentos. Universidad de Sonora. Luis Encinas y Rosales. Hermosillo, Sonora, C. P. 83000.

*Autor para correspondencia: amgarcia@guayacan.uson.mx
}

(Recibido mayo 2015; aceptado febrero 2016)

Palabras clave: estandarización, método analítico, espectroscopía

\section{RESUMEN}

Se evaluaron las condiciones analíticas ideales para cuantificar aluminio en tejidos de ratas Sprague Dawley, con la técnica de espectroscopia de absorción atómica por flama (EAAF). La importancia de estudios enfocados en el efecto del aluminio (Al) en los tejidos se debe a que recientemente ha sido considerado potencialmente tóxico ya que se le ha relacionado con alteraciones a nivel hepático, cerebral, hematológico y neoplásico, entre otras. Sin embargo, estos estudios no son concluyentes y aún se ignoran muchas de sus implicaciones. Para el presente trabajo los parámetros de calidad utilizados fueron: linealidad, exactitud (porcentaje de recuperación, \% Rec), precisión (porcentaje de coeficiente de variación, \% CV), límites de detección (LD) y de cuantificación (LC). Se digirieron muestras de ratas Sprague Dawley (mama, vísceras y hueso), además de muestras de agua y alimento, las cuales fueron fortificadas con 100 $\mathrm{mg} / \mathrm{L}$ de Al. Asimismo, se utilizó un material de referencia certificado que contiene $700 \pm 4 \mathrm{mg} / \mathrm{L}$ de $\mathrm{Al}$ (CRM-S-B, US) para evaluar la precisión y exactitud del método analítico. El resultado obtenido para la linealidad, a través de curvas de calibración para Al fue $\mathrm{r}=0.9998$; para la exactitud, evaluada como porcentaje de recuperación utilizando concentraciones de $\mathrm{Al}$ de 20,50 y $100 \mathrm{mg} / \mathrm{L}$, fue de $101 \%$, $98 \%$ y $97 \%$, respectivamente. Mientras que para la precisión, el \% CV fue de 2.15, 1.05 y 1.16 $\%$, respectivamente. Tanto la exactitud como la precisión se consideran parámetros aceptables ya que el criterio de aceptación es de $100 \pm 15 \%$ para el primero y $\mathrm{CV} \leq$ $2 \%$ para el segundo, con métodos instrumentales. El resultado del LD a partir de un estándar de alta pureza de $\mathrm{Al}$ fue de $0.06 \mathrm{mg} / \mathrm{L}$ y del LC fue de $0.19 \mathrm{mg} / \mathrm{L}$, los cuales son similares a los reportados en la literatura científica. Respecto a las muestras de ratas Sprague Dawley, los resultados de exactitud obtenidos fueron: agua (103\%), alimento $(99.3 \%)$, mama (100.9\%), vísceras (103.5\%) y hueso (100.6\%). Sobre el material de referencia certificado (CRM-S-B) que contiene $700 \mathrm{mg} / \mathrm{L}$ de $\mathrm{Al}$, se obtuvo una 
recuperación de $100.62 \%$, que se considera aceptable ya que el criterio de aceptación es de $100 \pm 5 \%$ para este tipo de materiales certificados, en tanto que la precisión fue de $0.61 \%(C V)$. Se concluye que el proceso de optimización de las condiciones analíticas para cuantificar Al en muestras de ratas Sprague Dawley mediante la técnica de EAA por flama es aceptable en muestras fortificadas con Al, y a partir de un estándar de referencia certificado.

Key words: standardization, analytical methods, spectroscopy

\begin{abstract}
The ideal analytical conditions for the quantification of aluminum in tissue of Sprague Dawley rats by using flame atomic absorption spectroscopy (FAAS) were evaluated. The significance of studies focusing on the effect of aluminum (Al) in tissue is based on recent findings that relate $\mathrm{Al}$ to brain, liver, hematologic, and neoplasic conditions; and consequently, $\mathrm{Al}$ is now rendered as being potentially toxic. Nevertheless, these studies are not conclusive and many of Al possible effects are still ignored. The quality parameters used in this work were: linearity, accuracy (recovery percentage), precision (variation coefficient percentage), and detection (DL) and quantification limits (QL). Tissue samples of Sprague Dawley rats were digested (breast, viscera and bone), along with water and food samples. Al samples were fortified by adding $100 \mathrm{mg} / \mathrm{L}$ of Al. In addition, a certified reference material that contains $700 \pm 4 \mathrm{mg} / \mathrm{L}$ of $\mathrm{Al}$ was used (CRM-S-B) to assess the accuracy and precision of the analytical method. The value for linearity, as obtained from the use of Al calibration curves, was $r=0.9998$. The accuracy level, measured as a recovery percentage using Al concentrations of 20, 50 and $100 \mathrm{mg} / \mathrm{L}$, was 101, 98 and $97 \%$, respectively. Precision values, quantified as the coefficient variation percentage $(\% \mathrm{CV})$ were $2.15,1.05$ and $1.16 \%$, respectively. The obtained accuracy and precision results are considered acceptable as the acceptance criterion using instrumental methods is $100 \pm 15 \%$ for the former and $\mathrm{CV} \leq 2$ $\%$ for the later. The concentration levels obtained for DL and QL, using a high-purity standard, were of $0.06 \mathrm{mg} / \mathrm{L}$ and $0.19 \mathrm{mg} / \mathrm{L}$, respectively; these values are similar to those reported in the scientific literature. The accuracy results ( $\%$ of recovery) obtained for Sprague Dawley rat samples were: water $(103 \%)$, food $(99.3 \%)$, breast cancer $(100.9 \%)$, viscera $(103.5 \%)$ and bone $(100.6 \%)$. The $100.62 \%$ recovery obtained for the certified reference material CRM-S-B, containing $700 \mathrm{mg} / \mathrm{L}$ of aluminum, is considered acceptable as the acceptance criterion for this type of certified materials is $100 \pm 5 \%$. In addition, the accuracy level was of $0.61 \%(\mathrm{CV})$. It is concluded that the analytical conditions optimization process to quantify Al in samples of Sprague Dawley rats using the flame AAS technique is acceptable in Al fortified samples, and from a certified reference standard.
\end{abstract}

\section{INTRODUCCIÓN}

El aluminio (Al) es uno de los elementos metálicos más abundante en la naturaleza, ya que representa el $8 \%$ de los metales que conforman la corteza terrestre. La forma más común en la que se encuentra es asociado con minerales tipo bauxita (Krewski et al. 2007). Recientemente se ha propuesto el término de metales tóxicos, sobre la base de la alta solubilidad en agua y el potencial tóxico a la salud humana que puedan llegar a tener, es en este grupo que se encuentra el Al (Tchounwou et al. 2012). Estimaciones hechas recientemente en Estados Unidos, establecen que una persona adulta joven y de clase media consume alimentos y bebidas que contienen $\mathrm{Al}$ con una cantidad ingerida en promedio entre 105 y $150 \mathrm{mg}$ de Al/día. Esto significa un consumo frecuente de este metal en sus diferentes formas o presentaciones de manera rutinaria. Este factor ha hecho que el Al sea objeto de múltiples investigaciones debido a sus implicaciones en la salud (DHHS 2008). Algunos investigadores a nivel mundial, han enfocado sus proyectos de investigación en demostrar la posible participación del $\mathrm{Al}$ como agente genotóxico, que 
puede provocar inestabilidad genómica y ésta a su vez, puede estar directamente relacionada con la carcinogénesis (Di Virgilio et al. 2010).

Por ello, es necesario relacionar la concentración de $\mathrm{Al}$ ingerida con la presencia de alguna patología. La técnica para cuantificar metales como el Al, es la espectroscopia de absorción atómica. Actualmente en México no existe en la Norma Oficial Mexicana un método de referencia para evaluar la concentración de $\mathrm{Al}$ en fluidos biológicos humanos o de animales de experimentación mediante la técnica de espectroscopia de absorción atómica (EAA). El objetivo del presente trabajo fue optimizar las condiciones analíticas ideales para cuantificar $\mathrm{Al}$ en tejido de ratas Sprague Dawley, mediante espectroscopia de absorción atómica por flama.

\section{MATERIALES Y MÉTODOS}

\section{Preparación del material de laboratorio}

El material de laboratorio fue sometido a un estricto control sanitario para evitar la contaminación por elementos traza, siguiendo el método recomendado por la Norma Mexicana NMX-AA-115-SCFI-2001 (Secretaría de Economía 2001). Los materiales de trabajo de vidrio (pipetas serológicas y volumétricas, matraces volumétricos Pyrex, entre otros) se lavaron exhaustivamente con detergente líquido comercial para después ser sometidos a una solución de $\mathrm{HNO}_{3}$ al $20 \%$ (grado analítico 70\% marca Fermont) durante tres días. Posteriormente fueron enjuagados con agua desionizada. Finalmente el material se secó y fue guardado en recipientes de plástico con tapa hermética hasta su uso.

\section{Calibración de balanza analítica y material vo- lumétrico}

El material volumétrico (pipetas de $5.0 \mathrm{~mL}$ y matraces de $100.0 \mathrm{~mL}$ ) fue calibrado con el método gravimétrico (Ruiz-Morer 2006), a través de una balanza analítica marca Vibra modelo AF-R220E 220/0.0001g (Max/d), la cual previamente también fue calibrada con un equipo de masas patrón de laboratorio $(0.50 \mathrm{~g})$ marca Rice Like, certificadas por la Entidad Mexicana de Acreditación, A.C. (EMA)

\section{Calibración del equipo de absorción atómica (EAA)}

Para la cuantificación de Al se utilizó un equipo de espectroscopía de absorción atómica (EAA), PerkinElmer Modelo AAnalyst 400. Para ello se preparó un estándar de $\mathrm{Al}$ de $50 \mathrm{mg} / \mathrm{L}$, en el que la respuesta del instrumento debe leer 0.22 de absorbancia, bajo las condiciones de operación recomendadas por el proveedor (Perkin-Elmer 2012).

\section{Evaluación de parámetros analíticos en condicio- nes ideales mediante EAA por flama}

Los parámetros analíticos de calidad considerados son los siguientes (CENAM-EMA 2008):

Linealidad. Se estimó a través de curvas de calibración a partir de un estándar de Al de 1000 mg/L (AccuTrace reference standard). Las concentraciones de trabajo fueron de 10, 20, 50, 100 y $200 \mathrm{mg} / \mathrm{L} \mathrm{de} \mathrm{Al.}$ Para preparar las disoluciones de trabajo se tomaron 1, 2, 5, 10 y $20 \mathrm{~mL}$ del estándar de Al de $1000 \mathrm{mg} / \mathrm{L}$ y se colocaron en matraces volumétricos de $100 \mathrm{~mL}$ cada uno. Se les agregó $2 \mathrm{~mL}$ de $\mathrm{HNO}_{3}$ concentrado y $10 \mathrm{~mL}$ de $\mathrm{KCl}$ al $2 \%$, posteriormente se aforó a 100 $\mathrm{mL}$ con agua desionizada, todo esto por triplicado. Se determinó la concentración de $\mathrm{Al}$ en el equipo de EAA, se graficó la concentración contra la absorbancia y se estimó el coeficiente de correlación de Pearson (r). El criterio de aceptación es de $r \geq 0.995$ (Miller y Miller 2002). El coeficiente de correlación se determinó mediante la siguiente ecuación:

$r=\frac{n \sum x y-\sum x \sum y}{\sqrt{n \sum x^{2}-\sum(x)^{2} * n \sum y^{2}-\sum(y)^{2}}}$

Dónde: $n$ es el tamaño de muestra, $x$ es la concentración, $y$ es la absorbancia.

Exactitud. Se estimó mediante el porcentaje de recuperación (\% Rec) para lo cual se prepararon soluciones a concentraciones de 20,50 y $100 \mathrm{mg} / \mathrm{L}$ de Al, a partir de una solución reserva de $1000 \mathrm{mg} / \mathrm{L}$, con cinco réplicas y cada una por triplicado. Estas tres concentraciones representan los puntos intermedios de la curva de calibración. Las disoluciones se prepararon de la misma manera en que se explicó en la sección previa. El porcentaje de recuperación se determinó a través de la siguiente ecuación:

$\% \operatorname{Rec}=\frac{\text { valor medido }}{\text { valor teórico }} \times 100$

Según la Unión Internacional de Química Pura y Aplicada (IUPAC, por sus siglas en inglés) el criterio de aceptación es $\% \operatorname{Rec}=100 \pm 15$ (rango: 85-115) (Thompson et al. 2002)

Precisión. Se determinó mediante el porcentaje del coeficiente de variación (\% CV), para ello se prepararon soluciones de $\mathrm{Al}$ de 20, 50 y $100 \mathrm{mg} / \mathrm{L}$, 
a partir de una solución reserva de $1000 \mathrm{mg} / \mathrm{L}$ de $\mathrm{Al}$, con cinco réplicas y cada una por triplicado. $\mathrm{El} \% \mathrm{CV}$ se obtuvo mediante la siguiente ecuación:

$\% \mathrm{CV}=\frac{\text { desviación estándar }}{\text { promedio }} \times 100$

El criterio de aceptación es $\% \mathrm{CV} \leq 2 \%$ para métodos instrumentales (Miller y Miller 2002).

Límite de detección (LD) y límite de cuantificación (LC). Se obtuvieron a partir de las curvas de calibración. Para ello se estimó el valor de la ordenada al origen (b), la pendiente (m) y la desviación estándar (s), con estos datos se calculó el LD como la concentración del analito que proporcionó una señal igual a la del blanco más tres veces la desviación estándar de dicho blanco. El LC se determinó como la concentración del analito que proporcionó una señal igual a la del blanco más diez veces la desviación estándar de dicho blanco (Miller y Miller 2002). Las ecuaciones para LD y LC son las siguientes:

$\mathrm{LD}=\mathrm{yB}+3 \mathrm{sB}$

$\mathrm{LC}=\mathrm{yB}+10 \mathrm{sB}$

Donde:

$\mathrm{y}_{\mathrm{B}}=$ concentración del analito que proporcionó una señal igual a la señal del blanco

$3 \mathrm{~s}_{\mathrm{B}}$ o $10 \mathrm{~s}_{\mathrm{B}}=$ Tres o 10 veces la desviación estándar del blanco

Para el cálculo del LD se procedió de acuerdo con la NMX-AA-051-SCFI-2001 (Secretaría de Economía 2016), de la siguiente manera:

a) Obtención de los valores de absorbancia $Y_{i, n}$ de los puntos de la curva de calibración, donde el número de estándares (i), podrán estar en el intervalo $\leq$ $\mathrm{i} \leq 10)$ y el número de réplicas (n) pueden estar para EAA por flama entre $(3 \leq n \leq 5)$.

b) De cada serie de datos se obtuvo la desviación estándar $\left(\mathrm{S}_{\mathrm{i}}\right)$ y el promedio de la absorbancia $\left(\overline{\mathrm{Y}}_{\mathrm{i}}\right)$, con éstos dos últimos se calculó el coeficiente de variación $\left(\mathrm{CV}_{\mathrm{i}}\right)$.

c) Comprobación visual de la linealidad entre la absorbancia promedio $\left(\bar{Y}_{i}\right)$ y la concentración al graficar $\bar{Y}_{i}$ vs $X_{i}$.

d) Determinación del coeficiente de correlación lineal, mediante la ecuación (1).

Si $r \geq 0.995$ se acepta el valor del coeficiente de correlación.
Si se cumplen las condiciones de los incisos c) y d), se calcula la pendiente (b) y el punto de intersección (a) de la recta en el eje Y, por medio del método de los mínimos cuadrados:

$\hat{\mathrm{Y}}=\mathrm{mX}+\mathrm{b}$

Dónde: $\hat{Y}$ es la absorbancia de la muestra ya procesada; $\mathrm{m}$ es la pendiente (coeficiente de absortividad), y b es la ordenada al origen. Despejar x que es la concentración de la muestra procesada.

e) Cálculo de las desviaciones $S_{\mathrm{y} / \mathrm{x}}$ entre los valores obtenidos por medio de la línea recta $\left(\hat{\mathrm{Y}}_{\mathrm{i}}\right)$ y los valores experimentales $\left(\bar{Y}_{\mathrm{i}}\right)$.

f) Cálculo de los valores de absorbancia $\left(\hat{Y}_{i}\right)$ sustituyendo en la línea recta las concentraciones $\left(\mathrm{X}_{\mathrm{i}}\right)$

g) Con los datos anteriores se obtuvo $\mathrm{S}_{\mathrm{y} / \mathrm{x}}$

$S_{y / x}=\frac{\sum\left(\bar{Y}_{i}-\hat{Y}_{i}\right)^{2}}{n-2}$

Por definición $\mathrm{S}_{\mathrm{y} / \mathrm{x}}$ es igual a la desviación estándar del blanco $\left(\mathrm{S}_{\mathrm{B}}\right)$ y el término independiente de la línea recta (a) es igual a la absorbancia de dicho blanco $\left(\mathrm{Y}_{\mathrm{B}}\right)$.

h) Por último se aplicó la definición de LD (ecuación 4) o de LC (ecuación 5).

El LD de un analito se describe como aquella concentración que proporciona una señal en el instrumento significativamente diferente de la de una muestra "blanco" o "señal de fondo". El LD del método se calculó con la ayuda de la zona de la representación cercana al origen, y se utilizó tanto la pendiente como la ordenada al origen (Miller y Miller 2002).

\section{Exactitud y precisión a partir de un material de referencia certificado}

Para evaluar de manera más estricta las condiciones de optimización del método analítico se determinó la exactitud y la precisión a partir de una solución acuosa certificada (CRM-S-B, High-Purity Standards, Charleston, SC, US) que contenía $700 \pm 4$ $\mathrm{mg} / \mathrm{L}$ de Al y pureza de 99.964 a $99.999 \%$, realizando 5 réplicas y cada una por triplicado. La exactitud se determinó mediante el \% Rec y la precisión a través del \% CV, tal y como se describió anteriormente.

\section{Aplicabilidad del método}

Se cuantificó Al en muestras biológicas de ratas 
Sprague Dawley (mama, cerebro, hígado y hueso), agua y alimento, los cuales fueron fortificados con $100 \mathrm{mg} / \mathrm{L}$ del estándar de Al de $1000 \mathrm{mg} / \mathrm{L}$. Las muestras fueron previamente digeridas por el método de vía húmeda (ácido inorgánico concentrado más temperatura) tal y cómo se describe a continuación:

\section{a) Obtención de muestras biológicas de ratas Sprague Dawley}

Se utilizó un grupo de seis ratas hembra Sprague Dawley de $200 \pm 20 \mathrm{~g}$, las cuales fueron anestesiadas en cámara con halotano y posteriormente eutanizadas por dislocación cervical de acuerdo a lo establecido en la NOM-033-ZOO-1995 (SAGARPA 1995). Los tejidos se obtuvieron mediante disección con bisturí de acero inoxidable. Se realizó un compuesto de cada grupo de tejidos y con el mismo volumen de agua desionizada fueron macerados en licuadoras con aspas de acero inoxidable. Los materiales utilizados se lavaron y se desmineralizaron previamente de acuerdo a la NMX115-AA-SCFI-2001 (Secretaría de Economía 2001).

\section{b) Condiciones de bioterio}

Un grupo de seis ratas hembra Sprague Dawley, sin ningún tipo de tratamiento específico fueron mantenidas en condiciones estándar de bioterio, con ciclos de luz/oscuridad de $12 \mathrm{~h}$, humedad de 40 a $70 \%$, temperatura de 18 a $22{ }^{\circ} \mathrm{C}$, agua y alimento ad libitum, de acuerdo a lo que establece la NOM-062ZOO-1999 (SAGARPA 1999).

c) Descomposición de las muestras de tejidos de ratas Sprague Dawley, agua y alimento, fortificadas con Al

Para la descomposición de las muestras de mama, hígado, hueso y cerebro de ratas SpragueDawley, controles, agua y alimento, se prepararon tres réplicas de $0.500 \pm 0.05 \mathrm{~g}$. A las muestras y a los blancos se les agregaron $10 \mathrm{~mL}$ de estándar de $\mathrm{Al}$ en forma de $\mathrm{AlCl}_{3}$ de alta pureza (AccuTrace reference standard). Posteriormente, se adicionaron $10 \mathrm{~mL}$ de $\mathrm{HNO}_{3}$ concentrado (Suprapur Merck). Las muestras se calentaron a una temperatura de $120^{\circ} \mathrm{C}$ hasta reducir el volumen a $3 \mathrm{~mL}$. Se agregaron $3 \mathrm{~mL}$ de $\mathrm{H}_{2} \mathrm{O}_{2}$ al $30 \%$ (con pureza del 29 al $32 \%$, marca Fermont), se calentaron hasta reducir el volumen a aproximadamente $2 \mathrm{~mL}$ e inmediatamente después se agregó $1 \mathrm{~mL}$ de $\mathrm{HCLO}_{4}$ concentrado. Finalmente se adicionaron $5 \mathrm{~mL}$ de $\mathrm{HNO}_{3}$ concentrado hasta la total mineralización de las muestras (Gómez-Álvarez 2004). El extracto ácido se llevó a un volumen de 100 $\mathrm{mL}$ con agua desionizada (la concentración final de $\mathrm{Al}$ es de $100 \mathrm{mg} / \mathrm{L}$ ).

\section{RESULTADOS Y DISCUSIÓN}

\section{Calibración de balanza analítica y material vo- lumétrico}

En el cuadro I se presentan los resultados de la calibración de la balanza analítica y el material volumétrico, en donde se puede apreciar que dicha calibración es aceptable (CENAM-EMA 2008).

CUADRO I. RESULTADOS DE LA CALIBRACIÓN DE LA BALANZA ANALÍTICA CON MASAS PATRÓN Y MATERIAL VOLUMÉTRICO POR TRIPLICADO

\begin{tabular}{lccc}
\hline Réplica & $\begin{array}{c}\text { Balanza } \\
\text { analítica } \\
\text { (masa 0.50 g) }\end{array}$ & $\begin{array}{c}\text { Pipeta } \\
\text { volumétrica } \\
(5.0 \mathrm{~mL})\end{array}$ & $\begin{array}{c}\text { Matraz } \\
\text { volumétrico } \\
(100.0 \mathrm{~mL})\end{array}$ \\
\hline 1 & 0.4990 & 4.9862 & 99.7766 \\
2 & 0.4990 & 4.9988 & 99.6352 \\
3 & 0.4991 & 4.9894 & 99.6479 \\
4 & 0.4991 & 4.9862 & 99.6682 \\
5 & 0.4989 & 4.9878 & 99.7091 \\
6 & 0.4990 & 5.0026 & 99.6730 \\
7 & 0.4991 & 4.9867 & 99.6917 \\
8 & 0.4990 & 5.0115 & 99.7447 \\
9 & 0.4991 & 4.9954 & 99.8136 \\
10 & 0.4990 & 4.9939 & 99.8489 \\
Promedio & 0.4990 & 4.9939 & 99.7209 \\
Desviación Estándar & 0.0001 & 0.0154 & 0.1894 \\
\hline
\end{tabular}

\section{Evaluación de parámetros analíticos en condicio- nes ideales mediante EAA por flama}

Linealidad. En la figura 1 se muestra el resultado de linealidad, el cual fue significativo y aceptable, ya que se obtuvo un valor del coeficiente de correlación de $r=0.9998$, por arriba de $r \geq 0.995$ (CENAM-EMA 2009).

Exactitud. Los resultados obtenidos a partir de concentraciones de 20, 50 y $100 \mathrm{mg} / \mathrm{L}$ de $\mathrm{Al}$ fueron

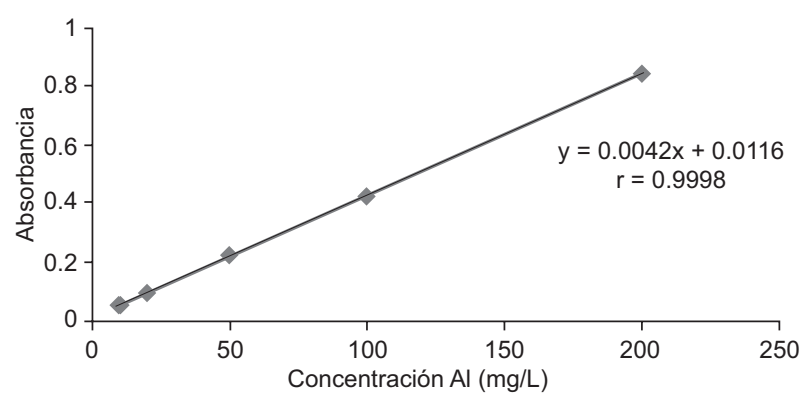

Fig. 1. Curva de calibración para aluminio (Al) mediante espectroscopía de absorción atómica por flama 
101, 98 y 97 \%, respectivamente (Cuadro II), los cuales se consideran dentro del rango recomendado de $100 \pm 15 \%$ por la Unión Internacional de Química Pura y Aplicada (IUPAC, por sus siglas en inglés) (Thompson et al. 2002) y por el Centro Nacional de Metrología (CENAM) y la EMA (CENAM-EMA 2008).

CUADRO II. RESULTADOS DE LA EXACTITUD Y DE LA PRECISIÓN PARA UN ESTÁNDAR DE ALUMINIO A TRES CONCENTRACIONES Y PARA UN MATERIAL DE REFERENCIA CERTIFICADO (CRM-S-B US), CUANTIFICADOS POR ESPECTROSCOPÍA DE ABSORCIÓN ATÓMICA POR FLAMA, POR TRIPLICADO

\begin{tabular}{lcrrr}
\hline Corrida & $\begin{array}{c}\mathrm{STD} \\
20 \mathrm{mg} / \mathrm{L}\end{array}$ & $\begin{array}{c}\mathrm{STD} \\
50 \mathrm{mg} / \mathrm{L}\end{array}$ & $\begin{array}{c}\mathrm{STD} \\
100 \mathrm{mg} / \mathrm{L}\end{array}$ & $\begin{array}{c}\mathrm{MRC} \\
700 \mathrm{mg} / \mathrm{L}\end{array}$ \\
\hline 1 & 20.32 & 49.00 & 92.82 & 707.00 \\
2 & 19.74 & 47.86 & 99.06 & 704.00 \\
3 & 20.00 & 49.68 & 100.12 & 702.00 \\
4 & 20.33 & 49.30 & 93.78 & 701.00 \\
5 & 20.11 & 49.50 & 100.46 & 708.50 \\
Promedio & 20.02 & 48.85 & 97.33 & 704.00 \\
DesvEst & 0.43 & 0.52 & 1.12 & 4.30 \\
\% REC & 101.00 & 97.68 & 97.33 & 100.62 \\
\% CV & 2.16 & 1.05 & 1.16 & 0.61 \\
\hline
\end{tabular}

STD $=$ estándar, $\mathrm{MRC}=$ material de referencia certificado, $\% \mathrm{REC}=$ porcentaje de recuperación, $\% \mathrm{CV}=$ porcentaje de coeficiente de variación, DesvEst $=$ desviación estándar

Precisión. Los resultados obtenidos del \% CV para las concentraciones de 20,50 y $100 \mathrm{mg} / \mathrm{L} \mathrm{de} \mathrm{Al}$ fueron de $2.16,1.05$ y $1.16 \%$, respectivamente (Cuadro II), los cuales están dentro de los criterios aceptados, que deben ser $\leq 2 \%$ (Miller y Miller 2002).

CUADRO III. PORCENTAJES DE RECUPERACIÓN PARA MUESTRAS DE TEJIDOS DE RATAS, AGUA Y ALIMENTO, FORTIFICADAS CON 100 $\mathrm{mg} / \mathrm{L}$ DE ALUMINIO, DIGERIDAS POR VÍA HUUMEDA Y CUANTIFICADAS MEDIANTE ESPECTROSCOPIA DE ABSORCIÓN ATÓMICA POR FLAMA, POR TRIPLICADO

\begin{tabular}{lcc}
\hline Muestra & $\begin{array}{c}\text { Control sin fortificar } \\
\text { con } \mathrm{Al}(\mathrm{mg} / \mathrm{L})\end{array}$ & $\begin{array}{c}\text { Muestra fortificada con } \\
\mathrm{Al}(\% \text { recuperación })\end{array}$ \\
\hline Mama & 0.97 & 100.90 \\
Hígado & 1.02 & 103.50 \\
Hueso & 0.87 & 100.60 \\
Cerebro & 2.33 & 120.50 \\
Agua & 1.03 & 107.00 \\
Alimento & 1.07 & 99.30 \\
\hline
\end{tabular}

LD y LC. El LD de Al fue de $0.0579 \mathrm{mg} / \mathrm{L}$, mientras que el LC fue de $0.1911 \mathrm{mg} / \mathrm{L}$. Estos resultados son similares a los reportados por la compañía Perkin Elmer (2012) y por Correia et al. (2006), aunque en los mencionados trabajos, utilizaron otro tipo de muestras biológicas. Una de las ventajas de utilizar métodos instrumentales, como la absorción atómica, es que son capaces de detectar y determinar cantidades de metales mucho más pequeñas que los métodos de análisis tradicionales. Esta ventaja permite apreciar la importancia de las concentraciones a nivel de trazas en muchos materiales, por ejemplo en muestras biológicas y ambientales, así se contribuye al desarrollo de otras técnicas en las cuales los límites de detección bajos son el mejor criterio para aplicarlas con éxito (Miller y Miller 2002).

\section{Descomposición de las muestras de tejidos de ratas Sprague Dawley, agua y alimento, fortifi- cadas con Al}

Los resultados, para determinar el porcentaje de recuperación de muestras fortificadas con Al, se muestran en el cuadro III. Se puede apreciar que los porcentajes de recuperación obtenidos fueron: mama (100.9\%), hígado (103.5\%), hueso (100.6\%), agua $(107.0 \%)$ y alimento $(99.3 \%)$, los que se encuentran dentro del rango de 85 a $115 \%$ como lo establece la IUPAC (Thompson et al. 2002). Esto indica que el método de descomposición utilizado en las muestras de rata Sprague Dawley fue adecuado para cuantificar Al, además de que no se pierde por volatilización y/o precipitación. Sin embargo, las muestras de cerebro están fuera del rango permitido (120.5\%), lo cual se puede deber a que las ratas que no fueron tratadas previamente con aluminio contienen y/o almacenan cantidades importantes de $\mathrm{Al}$ en el cerebro provenientes de otra fuente, por lo que se deberá determinar esto de manera objetiva y analítica.

\section{Exactitud y precisión a partir de un material de referencia certificado}

Como se puede apreciar en el cuadro II, el porcentaje de recuperación de $\mathrm{Al}$ obtenido fue de $100.62 \%$. Este valor se considera aceptable y se encuentra muy cercano al $100 \%$ de recuperación. El \% CV de la precisión fue de $0.61 \%$, por lo se considera aceptable debido a que para métodos instrumentales se acepta un valor menor de $2 \%$ (Thompson et al. 2002). Con base en los resultados obtenidos de los parámetros analíticos evaluados, es importante señalar que aún falta por evaluar estos mismos parámetros a través de espectroscopia de absorción atómica por horno de grafito, con la finalidad de analizar con cantidades 
de muestra muy pequeñas y/o con concentraciones de $\mathrm{Al}$ que no se pueden detectar por flama. Por otra parte, se sugiere realizar la descomposición de las muestras a través de horno de microoondas, que tienen una destacada eficiencia en el proceso de descomposición (Bohrer et al. 2008).

\section{CONCLUSIONES}

La optimización de las condiciones analíticas para cuantificar $\mathrm{Al}$ en condiciones ideales, así como en tejidos de ratas Sprague Dawley con la técnica de absorción atómica por flama, se considera aceptable. Sin embargo, se recomienda no sólo optimizar el método analítico, sino realizar su validación con un material de referencia certificado de matriz similar al tejido de ratas. Lo anterior con la finalidad de verificar la eficiencia (porcentajes de recuperación) del procedimiento analítico en una matriz real. Una vez hecho lo antes mencionado, se podrá estimar la incertidumbre del método analítico.

\section{AGRADECIMIENTOS}

Se agradece el apoyo y las facilidades proporcionadas por el Departamento de Ciencias Químico Biológicas y por el Departamento de Ingeniería Química y Metalurgia, de la Universidad de Sonora, para la realización del presente proyecto de investigación. Asimismo, se agradece la participación del Dr. Héctor Guzmán Grijalva, por la revisión y corrección de la redacción del resumen en inglés.

\section{REFERENCIAS}

Bohrer D., Dessuy M. B., Kaizer R., Nascimento P. C., Schetinger M. R. C., Morsch V. M., de Carvalho L. M. y García S. C. (2008). Tissue digestion for aluminum determination in experimental animal studie. Anal Biochem. 377 (2), 120-127.

DOI: 10.1016/j.ab.2008.03.029

CENAM-EMA (2008). Guía técnica sobre trazabilidad e incertidumbre en las mediciones analíticas que emplean las técnicas de espectrofotometría de absorción atómica y de emisión atómica con plasma acoplado inductivamente. Centro Nacional de Metrología-Entidad Mexicana de Acreditación, A.C. Guía técnica de laboratorio. México, D. F., México, 21 pp.

CENAM-EMA (2009). Guía de trazabilidad metrológica de los valores asignados a los calibradores y material de control empleados por el laboratorio clínico. Centro Nacional de Metrología-Entidad Mexicana de Acreditación, A.C. Guía técnica de laboratorio. México, D. F., México, 9 pp.

Correia L., Soares M. E. y Bastos M. L. (2006). Validation of an electrothermal atomization atomic absorption spectrometry method for the determination of aluminum, copper, and lead in grapes. J. Agric. Food Chem. 54 (25), 9312-9316. DOI: 10.1021/jf0620564

DHHS (2008). Public health statement aluminum. Division of Toxicology and Environmental Medicine. Program and Service. Department of Health and Human Services. Washington, EUA, 8 pp.

Di Virgilio A. L., Reigosa M., Arnal P. M., Fernández M. y de Mele L. (2010). Comparative study of the cytotoxic and genotoxic effects of titanium oxide and aluminium oxide nanoparticles in Chinese hamster ovary (CHOK1) cells. J. Hazard. Mater. 177 (1-3), 711-718. DOI: 10.1016/j.jhazmat.2009.12.089

Gómez-Álvarez A. (2004). Manual de métodos analíticos para rocas y minerales. Editorial Universidad de Sonora. Hermosillo, Sonora, México, 203 pp.

Krewski D., Yokel R. A., Nieboer E., Borchelt D., Cohen J., Harry J., Kacew S., Lindsay J., Mahfouz A. M., y Rondeau V. (2007). Human health risk assessment for aluminium, aluminium oxide, and aluminium hydroxide. J. Toxicol. Environ. Health B Crit. Rev. 10(Suppl1), 1-269. DOI: 10.1080/10937400701597766

Miller J.N. y Miller J.C. (2002). Estadística y quimiometría para química analítica. Cuarta edición. Pearson Prentice Hall. Madrid, España. 38 pp.

SAGARPA (1995). Norma Oficial Mexicana NOM-033ZOO-1995. Sacrificio humanitario de los animales domésticos y silvestres. Secretaría de Agricultura y Ganadería y Desarrollo Rural. Diario Oficial de la Federación. 18 de diciembre de 2014.

SAGARPA (1999). Norma Oficial Mexicana NOM-062ZOO-1999. Especificaciones técnicas para la producción, cuidado y uso de los animales de laboratorio. Secretaría de Agricultura y Ganadería y Desarrollo Rural. Diario Oficial de la Federación. 22 de agosto de 2001.

Secretaría de Economía (2001). Norma Mexicana NMXAA-115-SCFI-2001. Análisis de agua - Criterios generales para el control de la calidad de resultados analíticos. Secretaría de Economía. Diario Oficial de la Federación. 17 de abril de 2001.

Secretaría de Economía (2016). Norma Mexicana NMXAA-051-SCFI-2001. Análisis de agua. Determinación de metales por absorción atómica en aguas naturales, potables, residuales y residuales tratadas. Método de prueba. Diario Oficial de la Federación. 07 de diciembre de 2016. 
Perkin Elmer (2012). Manual de operación de equipo de espectroscopía de absorción atómica modelo AAnalyst 400. México, D. F., México, 98 pp.

Ruíz-Morer R. (2006). Recomendaciones para la calibración de material volumétrico en el laboratorio clínico. Química Clínica 25 (2), 104-110.

Tchounwou P. B., Yedjoum C. G., Patlolla A. K. y Sutton D. J (2012). Heavy metal toxicity and the environment. EXS. 101, 133-64.

DOI: $10.1007 / 978-3-7643-8340-4 \quad 6$
Thompson M., Ellison S.L.R y Wood R. (2002). International union of pure and applied chemistry technical report. Harmonized guidelines for single-laboratory validation of methods of analysis. Pure Appl. Chem. 74 (5), 835-855. 